\title{
Evolving Robots able to Self-localize in the Environment: The Importance of Viewing Cognition as the Result of Processes Occurring at Different Time Scales
}

\author{
Stefano Nolfi \\ Institute of Cognitive Science and Technologies \\ National Research Council (CNR) \\ Viale Marx, 15, 00137, Roma, Italy \\ e-mail: nolfi@ip.rm.cnr.it
}

\begin{abstract}
In this paper we address the problem of synthesizing mobile robots able to solve problems in which they cannot merely react to sensory input, but have to maintain an internal state as well. More precisely we will show how autonomous robots synthesized through an evolutionary process can solve problems that necessarily require an ability to integrate sensory-motor information over time. By presenting the result of a set of experiments in which evolving robots are asked to navigate and self-localize in the environment, we will show that successful results can be achieved by providing evolving individuals with neural controllers with neurons that (a) vary their activity at different rates to detect regularities at different time scales in the sensory-motor flow, and (b) use thresholded activation functions to detect events extending over time.
\end{abstract}

\section{Introduction}

A new research paradigm, that has been called Embodied Cognitive Science (Varela, Rosch, and Thompson, 1991; Brooks, 1991; Clark, 1997; Pfeifer and Scheier, 1999), has recently challenged the traditional view according to which intelligence is an abstract process that can be studied without taking into consideration the physical aspects of natural systems. In this new paradigm, researchers tend to stress (1) situatedness, i.e., the importance of studying systems that are situated in an environment (Brooks, 1991, Clark, 1997), (2) embodiment, i.e., the importance of study systems that have bodies, receive input from their sensors and produce motor actions as output (Brooks, 1991; Clark, 1997), and (3) emergence, i.e. the importance of viewing behavior and intelligence as the emergent result of fine-grained interactions between the control system of an agent including its constituents parts, the body structure, and the environment. An important consequence of this view is that the agent and the environment constitutes a single system, i.e. the two aspects are so intimately connected that a description of each of them in isolation does not make much sense (Marturana and Varela, 1980, 1987; Beer, 1995).

This new approach has been successfully applied to synthesize robots exhibiting a large variety of interesting behavior. Most of these behaviors, however, consist of forms of reactive behaviors (i.e. behaviors that can be produced by agents that do not integrate sensory-motor information over time but always react in the same way to the same sensory state) or behaviors in which internal mechanisms play a rather limited role (Keijzer, 2001; Nolfi and Marocco, 2001). The next goal is to develop agents able to solve more complex problems in 
which agents cannot be guided toward the goal by the continuous update state of sensors and need some internal states as an addition to the missing sensory signals (Keijzer, 2001) Given that internal states should be extracted and/or continuously updated on the basis of the continuous flow of sensory-motor states, these forms of behaviour require an ability to integrate sensory-motor information over time (Beer 1995, 1996, Nolfi and Marocco, 2001). Indeed, to produce such behaviour organisms should possess two abilities: (1) an ability to extract useful internal states by integrating sensory-motor information over time and/or an ability to continuously update such states, and (2) an ability to act appropriately by taking into account both the current sensory-motor and internal states.

In this paper we will show how the embodied cognitive science approach can be extended to successfully tackle problems that cannot be solved through simple reactive strategies but necessarily require an ability to integrate sensory-motor states over time. More precisely, we will show how autonomous robots synthesized through an evolutionary process (Nolfi and Floreano, 2000) can develop such complex skills in close interaction with the environment without human intervention.

As we will see, successful results can be achieved by providing evolving individuals with neural controllers with neurons that (a) vary their activity at different rates to detect regularities at different time scales in the sensory-motor flow, and (b) use thresholded activation functions to detect events extending over time. In the next three sections we will describe the experimental setup, the obtained results, and the general implications of the experiments performed

\section{The experimental setup}

To investigate the issues described above in an experimental framework we decided to try to evolve a robot able to travel along a loopy corridor (see Figure 1, left) and to identify its current location in the environmente

\footnotetext{
${ }^{1}$ Form of behaviors more complex than simple reactive behavior or problems that require solutions more complex than reactive solutions have been called representation-hungry problems by Clark (1997) and anticipatory behavior by Keijzer (2001). I prefer behaviors that require an ability to integrate sensory-motor information over time, a terminology introduced by Beer $(1995,1996)$. This term stresses the fact that useful internal states are not given but should be extracted by the agent and/or continuously updated on the basis of the continuous flow of sensory-motor states.

${ }^{2}$ It should be noted that discriminating between problems that can be solved through purely reactive behavior and more complex problems that necessarily require more complex solutions is far from trivial if not impossible (see Nolfi, 1999, Nolfi and Marocco 2001). In this case, the main data that supports the assumption that this problem cannot be solved through a simple reactive strategy is the fact that simple reactive controllers produce rather poor performance while controllers also relying on internal states display close to optimal performance (see below).
} 

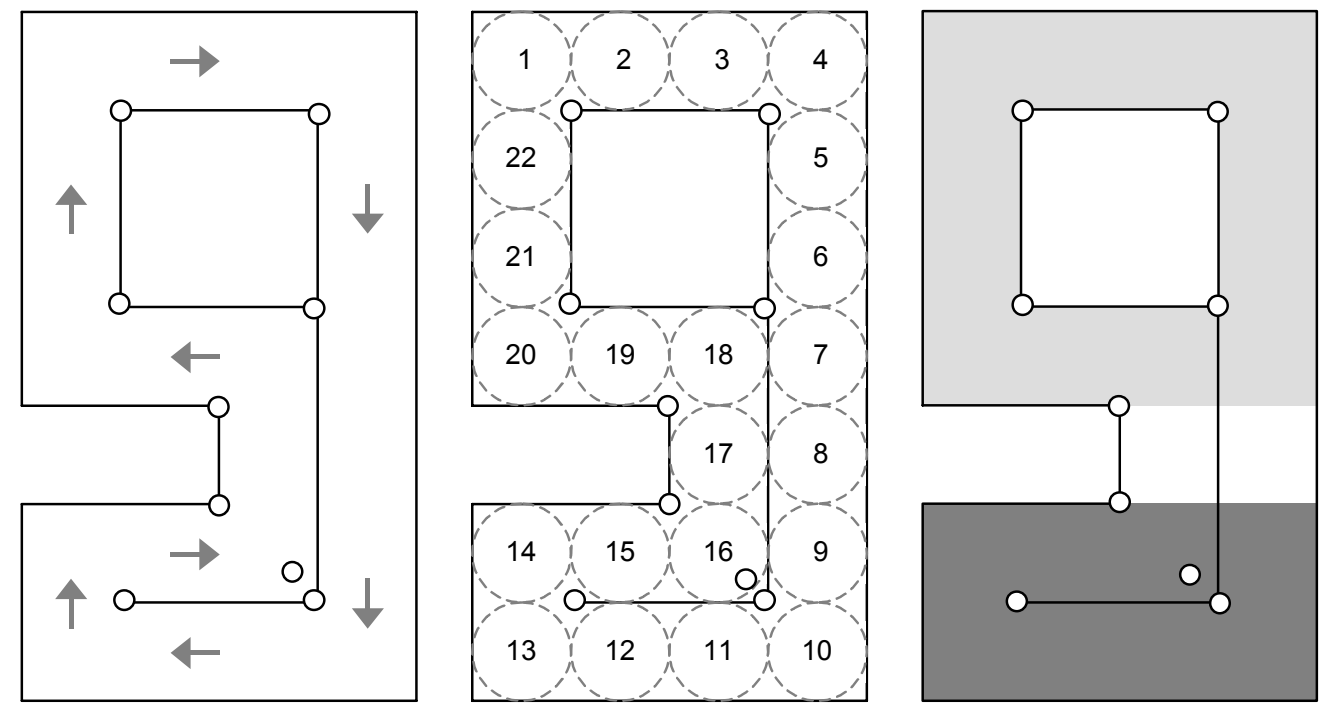

Figure 1. Left: The environment consists of a loopy corridor of $40 \times 70 \mathrm{~cm}$. Lines represent walls, circles represent cylindrical objects, and arrows represent the starting positions and orientations in which the robot is placed at the beginning of each trial. Center: The environment is divided into 22 idealized regions placed along the corridor clockwise. Right: The environment is also ideally divided into two rooms that are indicated in the Figure with light and dark gray colors.

The robot used in the experiments described here is Khepera (Mondada et al., 1993) a miniature mobile robot with a diameter of $55 \mathrm{~mm}$ and a weight of $70 \mathrm{~g}$ (Figure 2). It is supported by two lateral wheels that can rotate in both directions and two rigid pivots in the front and in the back. By spinning the wheels in opposite directions at the same speed, the robot can rotate without lateral displacement. The sensory system employs eight infrared sensors that are able to detect obstacles up to about four $\mathrm{cm}$. Experiments were conducted in simulation by using an extended version of Evorobot (Nolfi, 2000). To simulate the robot and the environment as accurately as possible, a sampling procedure was used to compute the activation state of the infrared sensors. Walls and cylindrical objects were sampled by placing one physical robot in front of them and by recording the state of the infrared sensors while the robot was turning 360 degrees at 20 different distances from of each object. These recorded values were used in simulation to set the activation states of the simulated infrared sensors on the basis of the current angle and distance of the robot with respect to obstacles. This procedure allows to develop a very accurate simulation that takes into account the detailed characteristics of the individual robot used in the experiments (Nolfi and Floreano, 2000).

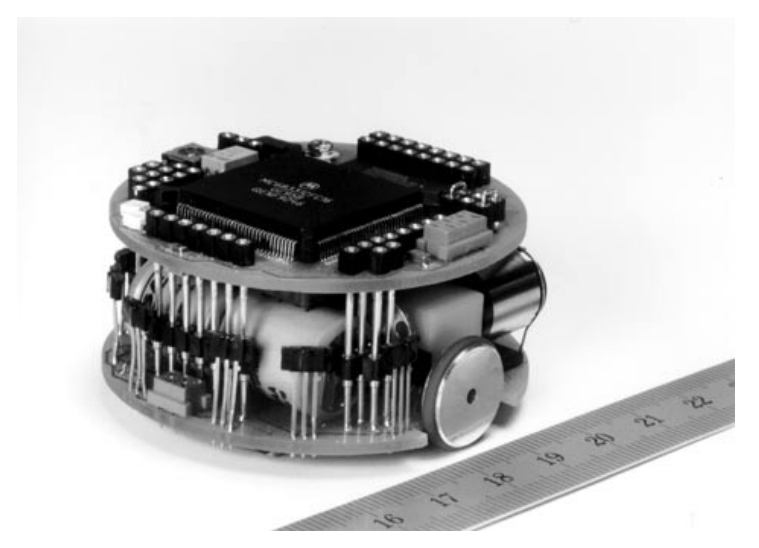

Figure 2: The Khepera robot. 
The controller of each individual consists of a neural network with nine sensory neurons directly connected to three motor neurons and five internal self-recurrent neurons receiving connections from the sensory neurons and sending connections to the motor neurons (see Figure 3). The first three sensory neurons encode the state of the three corresponding motor neurons at the previous time step, the other six sensory neurons encode the six frontal infrared sensors (normalized between [0.0, 1.0]). The first two motor neurons encode the desired speed of the two corresponding wheels and the last motor neuron encodes the robot's self-localization output (see below).

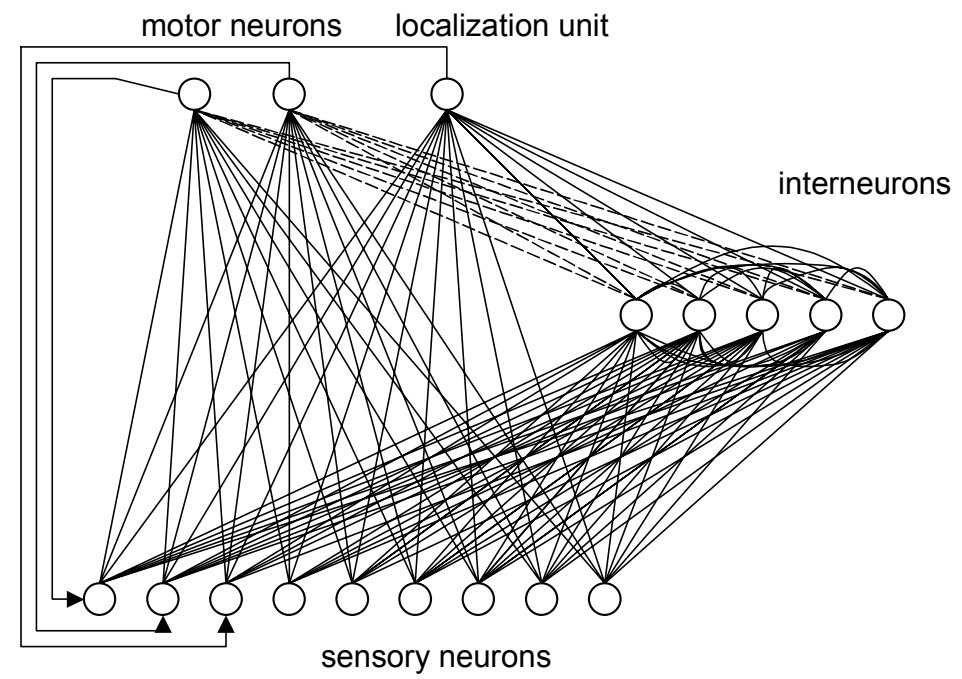

Figure 3: The architecture of the neural controller. The connections indicated with dashed lines (i.e. the connections between interneurons and the first two motor neurons that control the two wheels) are present only in some of the experiments (see text below).

By running a set of control experiments in which individuals were provided with reactive controllers that did not included internal neurons, we observed that evolved individuals were unable to solve the self-localization problem (result not shown). Indeed, the room in which the robot is located cannot be identified on the basis of the current sensory-motor information only, but can only be identified by taking into account the current and the previous sensorymotor states experienced by the robot. In other words, self-localization in this experimental setting is an example of a problem that necessarily requires an ability to integrate sensorymotor information over time.

During the evolutionary process the architecture is kept fixed. Only the biases and the time constants of neurons (i.e. the parameters that determine the rate of change of the activation state of neurons, see next section) and the synaptic strengths of the connections are encoded in the genotype and allowed to change. All parameters are encoded in the genotype with eight bits. Connection weights and biases are then normalized in the range [-10.0, 10.0], time constants are normalized in the range $[0.0,1.0]$. The initial population consists of 100 randomly generated genotypes. Each individual of the population is allowed to leave for eight epochs consisting of 2500 lifecycles each (each lifecycle lasts $100 \mathrm{~ms}$ ). At the beginning of each epoch the robot is initially placed in the eight corresponding positions and orientations indicated with the arrows in the left of Figure 1. Orientations are chosen to follow the clockwise direction of the corridor, however, a randomly selected angle in the range $[-10,+10]$ is in each trial added to the initial orientation of the robot. The 20 fittest individuals of each generation are allowed to reproduce by generating five copies of their genotype with $2 \%$ of their bits replaced with a new randomly selected value. The process is 
repeated for 500 generations. Each replication last about 1.5 hours on a standard $2 \mathrm{GHZ}$ PC and would take about three years on the real robot if testing one individual at a time.

The fitness function has two components that reward, respectively, the ability to travel clockwise along the corridor and the ability to indicate the current position of the robot in the environment. The first component is calculated by virtually dividing the environment in 22 adjacent regions (see Figure 1, center) and by computing the number of times a robot moves from one region to the next during its lifetime. The number of visited regions is truncated to 1000 (i.e. visiting more that 1000 regions does not provide any additional advantage) and normalized in the range $[0.0,1.0]$. The second component is calculated by virtually dividing the environment in two rooms, a dark and a light gray room, and by computing the percentage of times in which the robot correctly self-localizes in the two rooms (i.e. the fractions of lifecycles in which, while the robot is situated in the dark gray room, the activation of the robot's self-localization unit is lower than 0.5 , and the fraction of lifecycles in which, while the robot is situated in the light gray room, the activation of the robot's selflocalization unit was higher than 0.5 ). The second component is computed only if the number of visited regions is equal or greater than 1000. If the number of visited regions is lower than 1000, the total fitness is computed as follows: $\Phi=\frac{C}{1000}$, where $C$ is the number of visited regions while, if the number of visited regions is equal or greater than 1000 , the total fitness is computed as follows: $\Phi=1+\left(L^{*} D\right)$, where $L$ and $D$ are the percentage of times in which the robot correctly localizes itself in the light and in the dark gray rooms respectively. This implies that optimal performance correspond to fitness values that approximate 2.0 (this maximum value cannot be fully reached given that robots necessarily produce incorrect selflocalization answers during the very first part of each trial until they are able to self-localize). The rationale behind this fitness function is that evolving individuals should first acquire an ability to travel clockwise along the corridor and later acquire an ability to also self-localize in the environment.

\section{Results}

By running evolutionary experiments we observed that the ability to self-localize only emerges if internal neurons of evolving individuals are updated on the basis of activation functions that are suited to extract regularities at different time scales and to detect regularities that extend over a given amount of time. Figure 4 summarizes the results of two set of experiments in which: (a) internal and motor neurons are updated according to the logistic function (see equation 1), and (b) motor neurons are updated according to the logistic function but internal neurons are updated according to equation 2 .

$$
\begin{gathered}
O_{j}=\left(1+e^{-A_{j}}\right)^{-1} \\
O_{j}=\left\{\begin{array}{l}
\sqrt[2]{\tau_{j}} O_{j}^{(t-1)}+\left(1-\sqrt[2]{\tau_{j}}\right)\left(1+e^{-A_{j}}\right)^{-1} A_{j} \geq 0 ; \\
0 \text { otherwise }
\end{array}\right.
\end{gathered}
$$

With $O j^{(t-1)}$ being the output of the $j$ th neuron at the previous time step, $A j$ being the activity of the $j$ th neuron, $\tau j$ the time constant of the $j$ th neuron. 
In all cases, the activity of sensory neurons correspond to the activation state of the corresponding infrared sensor (normalized in the range $[0.0,1.0]$ ) or to the output of the corresponding motor neuron, and the activity of internal and motor neurons is computed according to the following function:

$$
A_{j}=t_{j}+\sum w_{i j} O_{i}
$$

With $t j$ being the bias of the $j$ th neuron, $W i j$ the weight from the $i$ th to the $j$ th neuron, $O i$ the output of the $i$ th neuron that sends a connection to the $j$ th neuron. All neurons are updated each $100 \mathrm{~ms}$.
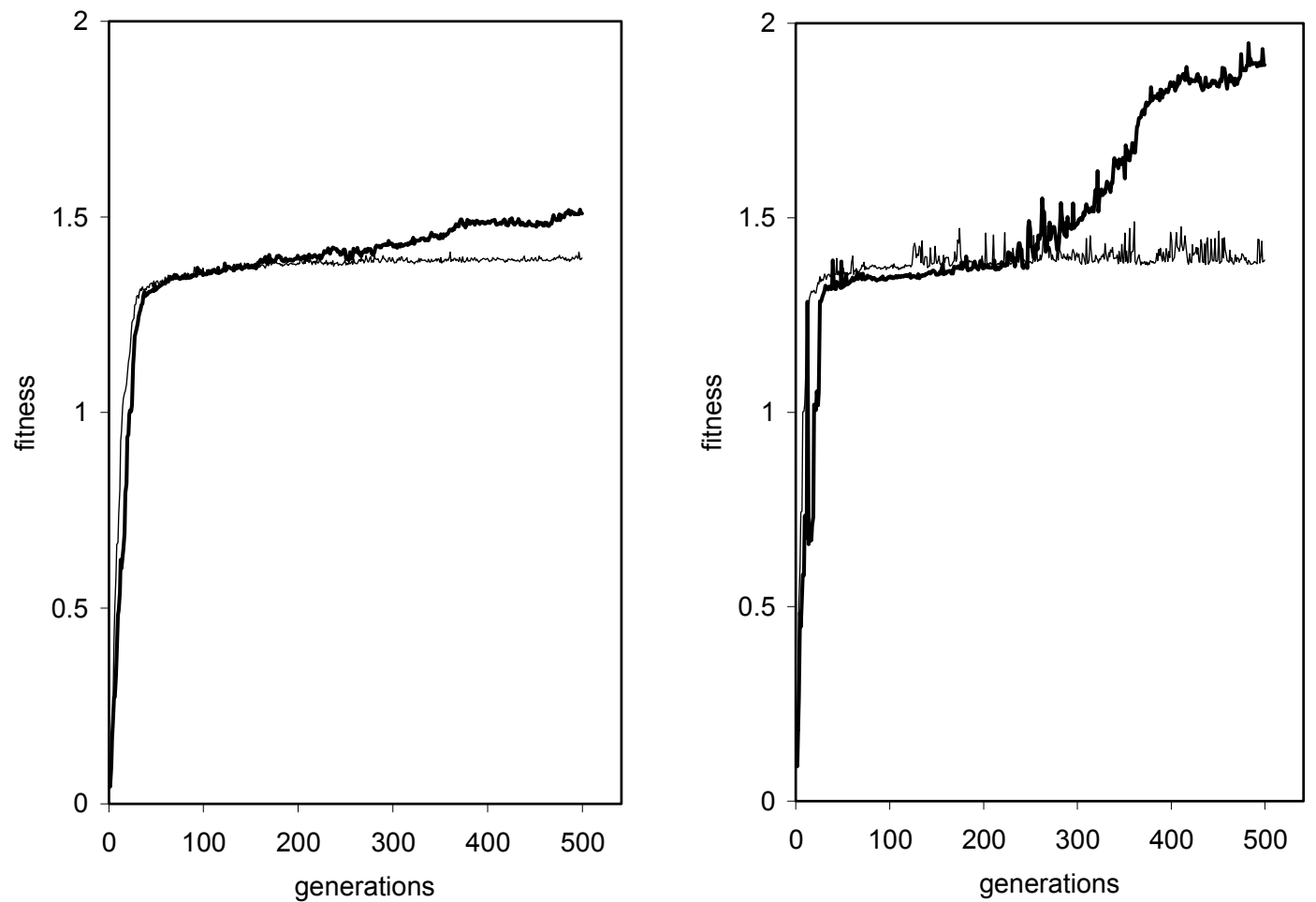

Figure 4. Fitness throughout generations of the best individual of each generation. Thin and thick lines represent performance achieved by individuals whose internal neurons are activated according to equation 1 and 2 respectively. Left: Average result of 10 replications. Right: Result of the best replication for each experimental condition.

As shown in Figure 4, during the first 20 generations the fitness of evolving individuals increases rather quickly and reaches value around 1.25 in both experimental conditions. At this stage evolving individuals maximize performance with respect to the first fitness component (the component that rewards the ability to travel fast along the corridor) but produce chance level performance with respect to the second fitness component (the components that rewards the ability to self-localize). During the successive evolutionary phase, the individuals of the second experimental condition (in which internal neurons are updated according to equation 2) clearly outperform the individuals of the first experimental condition (in which internal neurons are updated according to equation 1) At the end of the

\footnotetext{
${ }^{3}$ In running another set of experiments (results not illustrated in details) in which internal neurons were updated according to a variation of equation 2 in which the output of the neuron was not reset when the activity was
} 
evolutionary process in fact, the best individuals of the second experimental condition produce higher performance than the best individuals of the first experimental condition on the average (Figure 4, left). Moreover, while the best individuals of the second experimental conditions display close to optimal performance (i.e. correct self-localizations in at least $90 \%$ of the cases) in two out of ten replications, the individuals of the first experimental conditions always produce close to chance level performance with respect to the self-localization ability (Figure 4, right).

To explain the difference in performance between the two sets of experiments we should consider that equation 2 allows neurons to easily detect events that extends over time. This can be easily demonstrated by analyzing the activation state of internal neurons in evolved individuals. As can be seen in Figure 5, the activation of the i3 internal neurons (that has a time constant of about 0.99 and therefore tends to change its activation state rather slowly) its reset to 0 when the left-side infrared sensor (s4) is almost fully off (when the robot crosses the corner indicated with the letter $\mathbf{A}$ ). After this point, the activation state of this neuron keeps growing and reaches the maximum activation level after point $\mathbf{B}$. This information (i.e. the time the robot has spent traveling along the corridor after crossing the corner A) allows the robot to easily determine the room in which it is currently located. In fact, the robot is always located in the light or in the dark gray room when the output of internal neuron s3 is below or above a given threshold respectively. The fact that this internal neuron is reset only when the robot crosses corner A is due to the fact that, given the particular way in which this robot travels along the corridor (i.e. the fact that the robot closely follows the left wall) and given the characteristics of this particular environment, the left-side infrared sensor (s4) is almost fully off only when the robot travel along this particular location of the environment.

In principle, the same information computed by a single neuron updated according to equation 2 could be computed by a simple neural circuitry involving few neurons updated according to equation 1 and few connections with appropriate connection strengths. The difference in performance between the two set of experiments, however, suggests that the adoption of equation 2 significantly increases the evolvability of these systems (i.e. the probability that the application of the genetic operators, by modifying the free parameters of the individuals, can produce progressively better solutions).

A

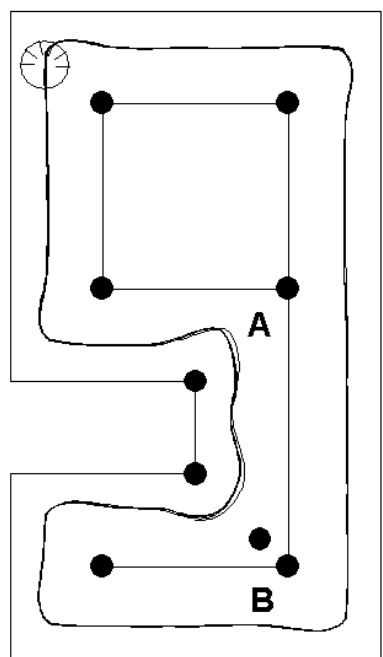

B

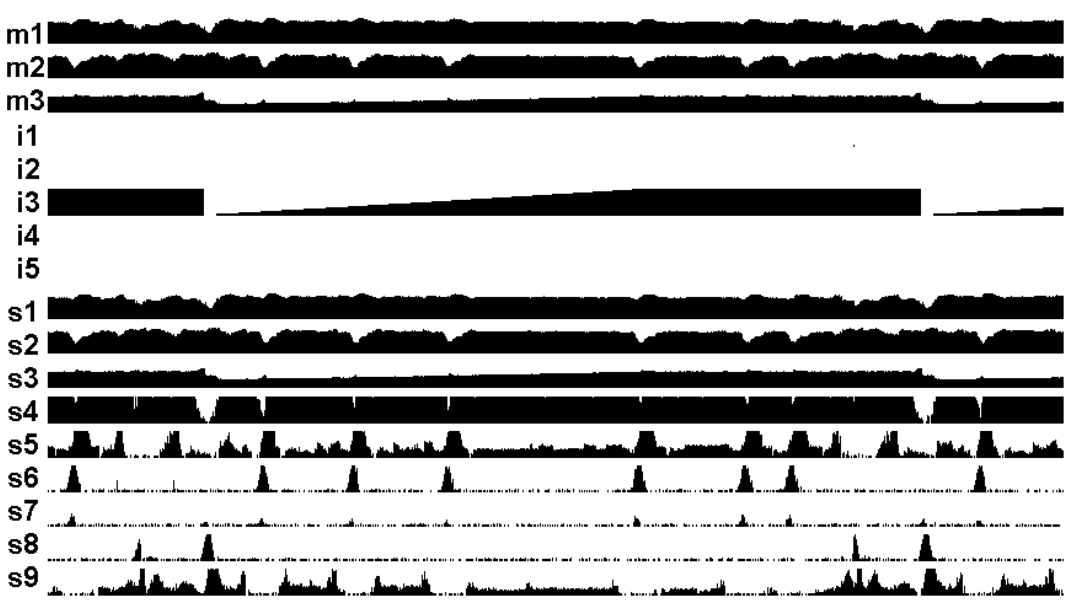

lower than 0 we did not observe the emergence of individuals able to solve the self-localization problem.

Performance was very similar to that shown in Figure 4, thin line. 
Figure 5. Neural outputs of a successful evolved robot traveling in the environment. Left: The environment and the robot trajectory during a few laps of the corridor. A and $\mathbf{B}$ indicate the approximate positions of the robot when the output of the third internal unit is reset and when it reaches the maximum value, respectively. Right: The output value of motor (m1-m3), internal (i1-i5), and sensory (s1-s9) neurons while the robot is traveling along the corridor (the output value is represented by the height with respect to the baseline).

\subsection{Extracting regularities over time at different levels of organization}

Another interesting aspect to investigate is whether providing also the sensory neurons with a time constant parameter that determine their rate of change could be advantageous. To investigate this issue, we ran a new set of experiments in which internal and motor neurons are activated according to equation 1 and 2 respectively (as in the previous experiment described above) but sensory neurons are activated according to the following equation:

$$
O_{j}=\tau_{j} O_{j}^{(t-1)}+\left(1-\tau_{j}\right) S_{j}
$$

With $O j$ being the output of the $j$ th neuron, $O j^{(t-1)}$ the output of the same neuron at the previous time step, $\tau j$ the time constant of the $j$ th neuron, $S j$ being the state of the corresponding sensor (normalized in the range $[0.0,1.0]$ ) or the state of the corresponding motor neuron at the previous time step (in the case of the three sensory neurons that encode the state of the three motor neurons at the previous time step). Notice that, as in the case of the internal neurons updated according to equation 2, the time constant parameter determines how much the activation state of the neuron is influenced by its previous activation state or, in other words, how slowly the neuron tends to change its activation state over time. Contrary to the internal neurons updated according to equation 2, these sensory neurons are never reset to 0 .

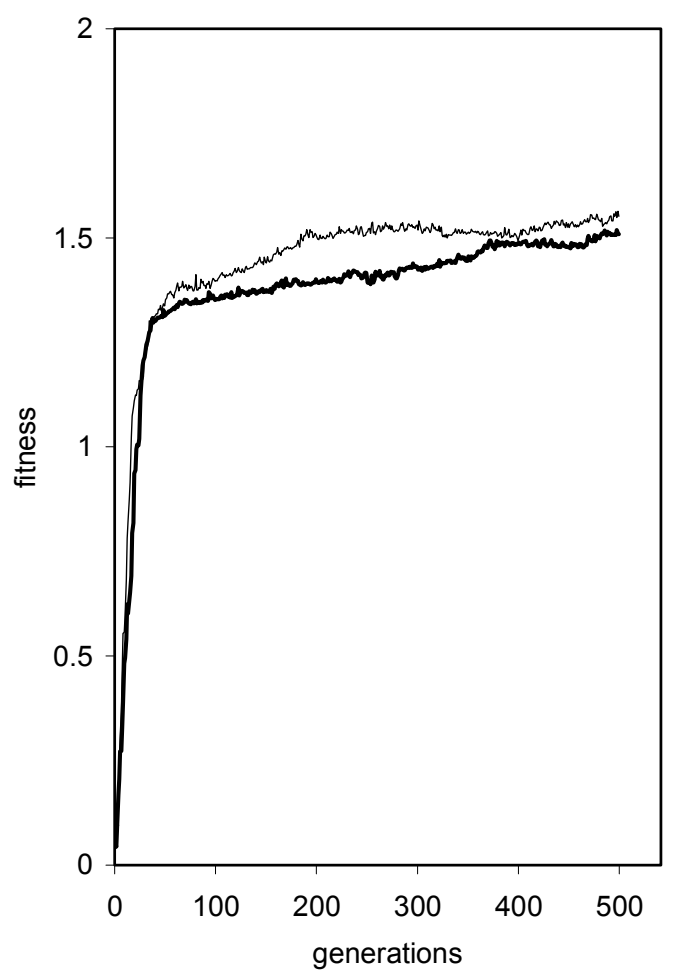


Figure 6. The thick line represents the same data shown in Figure 4, left. The thin line represents the results of a new experiment in which sensory neurons are updated according to equation 4. Average results of ten replications for each experimental condition.

As shown in Figure 6, neural controllers in which sensory neurons are activated according to equation 4 (thin lines) slightly outperform neural controllers in which sensory neurons encode the current state of the infrared or motor neurons (thick lines), on the average. Close to optimal performance (i.e. correct self-localizations in at least $90 \%$ of the cases) was observed in two out of ten replications in both experimental conditions.

Interestingly, sensory neurons updated according to equation 4 are able to detect regularities occurring at short time scales, and internal neurons, capitalizing on that, are used to detect higher level regularities occurring at longer time scales. As shown in Figure 7, displaying the behavior and the neural activity of one of the most successful individuals, the internal neuron il is turned off when the robot negotiates corners (see the locations indicated with the letter $\mathbf{A}$ on the left side of the Figure) and increases its output while the robot travels along a straight corridor. Thanks to a recurrent positive connection, however, the neuron is turned off on corners only if its activation level is below a given threshold or when the robot negotiates the narrow passage indicated with the letter $\mathbf{C}$. The final result is that this neuron is always below a given threshold in the light gray room due to the reset of its activity occurring in $\mathrm{C}$ and in A corners and is always over that threshold in the dark gray room.

As in the case of the previous strategy described in Figure 5, internal neurons are used to capture sensory-motor regularities that extend over rather long time scales (ranging from few to several seconds). In this experiments however, the high scale regularities extracted by internal neurons are based on short scale regularities, such as corners or narrow passages, ranging from few to several hundreds of milliseconds that are extracted by sensory neurons. Indeed, the internal neuron $\mathrm{il}$ has a time constant of 0.98 (i.e. it tends to change its activation state very slowly) while sensory neurons have time constants that range from 0.04 to 0.44 (i.e. they tend to change their activation state much more quickly). This allows evolving individuals to solve the self-localization problem even when no single sensory state uniquely identifies a given location of the environment and the whole problem can only be solved by extracting regularities at different time scales.
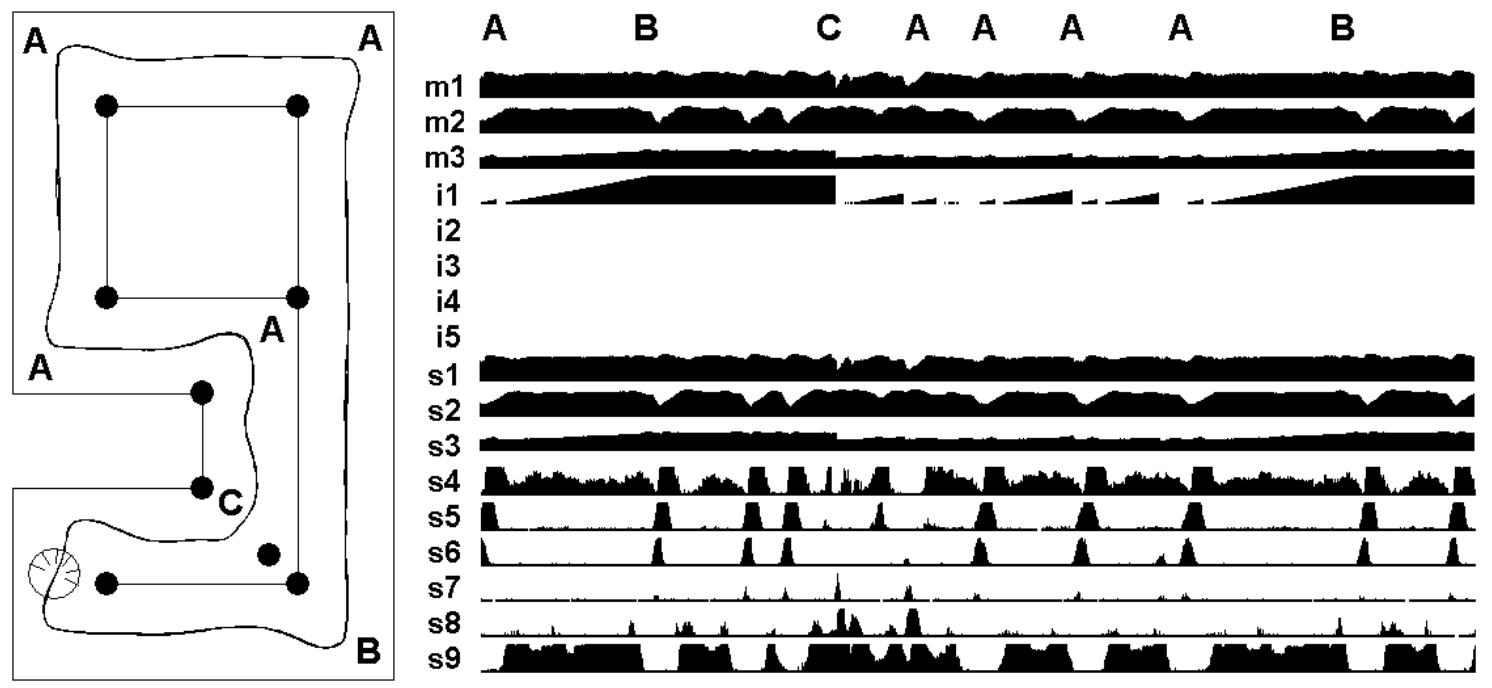

Figure 7. Neural outputs of a successful evolved robot traveling in the environment. Left: The environment and the robot trajectory during a few laps of the corridor. $\mathbf{A}$ and $\mathbf{C}$ indicate the approximate positions of the robot when the output of the first internal unit is reset to 0 . $\mathbf{B}$ indicates the position of the robot when the first internal unit reaches its maximum activation level. Right: The output value of motor (m1-m3), internal (i1-i5), and 
sensory (s1-s9) neurons while the robot is traveling along the corridor (the output value is represented by the height with respect to the baseline).

\subsection{Interference between the development of different abilities}

Finally, we investigated whether the development of the ability to self-localize does interfere with the previous acquired ability to travel along the corridor (remember that evolving individuals are rewarded for the ability to self-localize only if they are also able to effectively travel along the loopy corridor). To verify if this interference really occurs we ran a new set of experiments in which the connections from the internal neurons to the first two motor neurons controlling the two wheels (shown with dashed lines in Figure 3) were absent. The elimination of such connections results in neural controllers organized into two independent neural modules: a first neural module (constituted by the connection weights between the sensory neurons and the first two motor neurons controlling the two wheels) that controls the motion of the robot in the environment, and a second neural module (constituted by the connection between the sensory neurons, the internal neurons, and the last output units encoding the self-localization output) that is responsible for the ability to self-localize. As in the case of the experiment described above, sensory, internal and motor neurons were updated according to equation 4, 2 and 1 respectively.

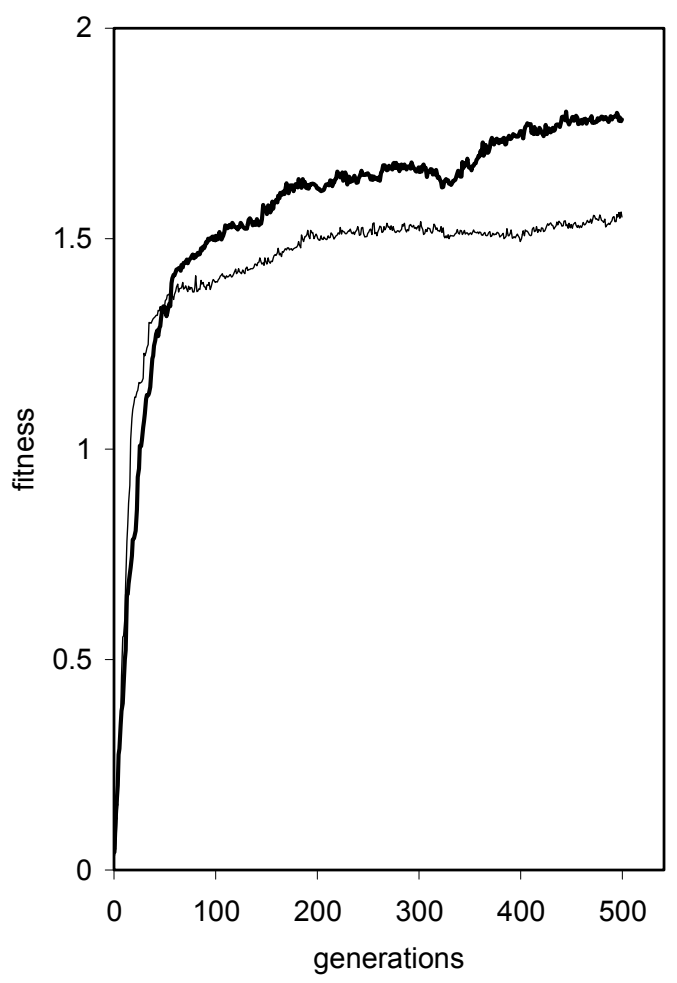

Figure 8. Thin and thick lines represent the fitness throughout generations obtained by providing or not providing the neural controllers with the connection from the internal neurons to the first two motor neurons controlling the two wheels respectively. Results corresponding to the thin line are the same results shown in Figure 6. Average results of 10 replications for each experimental condition.

As shown in Figure 8, when removing the connection between internal neurons and the first two motor neurons controlling the two wheels, significant better performance was achieved on average. Close to optimal performance was achieved in nine out of ten replications while in the experiments described above they were achieved only in two out of ten replications. This can be explained by considering that mutations affecting internal neurons in individuals provided with full connectivity between internal and motor neurons, 
by potentially affecting the way in which the robot travels in the environment and not only the self-localization output, have a much higher probability of being counter-adaptive. Indeed, changes affecting the internal neurons either might cause the loss of the ability to travel fast along the corridor or, by modifying the way in which the robot moves in the environment, might make it impossible to extract sensory-motor regularities over time that were used by individuals of previous generations to solve, at least in part, the self-localization problem.

\subsection{Spiking and dynamical neural networks}

Before concluding this section we will discuss the relation between the activation functions described above and the other related models discussed in the literature. Biological neurons communicate by sending pulses (spikes) along the axons to other neurons that alter the electric voltage of the membrane of the postsynaptic neurons. A neuron produces a spike when the electric potential of its membrane exceeds a given threshold and then needs some time to re-establish its electrochemical equilibrium before being able to generate a new spike. In the majority of the cases, computational models of neural networks assume that what matters in the communication among neurons is the firing rate and that this quantity can be conveniently represented by the activation level of the neuron as in equation 1 . Unfortunately, this form of simplification, does not only neglects the possible importance of the precise time of emission of a single spike (Villa, 2000), but also neglect the fact that real neurons are dynamical systems, i.e. their activation state is partially influenced by their previous activation state. To overcome the latter simplification one should rely on slightly more complex models such us spiking neural networks in which the single spikes are modeled (Rieke et al., 1997; Floreano and Mattiussi, 2001) or dynamical neural networks in which, as in equation 2 and 4, a time constant parameter is associated to the passive property of neurons (Hopfield, 1984; Beer and Gallagher 1992).

The specific activation functions described in this paper have not been directly inspired by biological evidences. Interestingly, however, internal neurons updated according to equation 2 and 4 resemble the slowly discharging spiny neurons of the striatum that produce outputs that last between 100ms and several seconds (Schultz et al., 1995). Interestingly, "In most cases, the activity of striatal neurons is not sufficiently explained by the physical characteristics of the stimuli presented or the movements performed but depends on certain behavioral situations, certain conditions, or particular kinds of trials in a given task, thus showing the relationships to the context in which the particular events occurred" (Schultz et al., 1995, p.12).

\section{Discussion}

Dealing with the real world or realistic simulations necessarily implies to deal with events that extend over a wide range of time scales. Regularities of this sort can only be detected by considering amount of changes in amount of time (Van Gelder and Port, 1995) and by choosing the appropriate time scale. As reported by Keijzer (2001), the picture-book Powers of Ten of Philip and Phylis Morrison is a clear example of the relation between the scale chosen and the regularities that can be detected (in the context of space instead of time). The book is a visual journey consisting of 42 images, ranging from the entire known universe to three quarks with a proton, where each image portrays a part of the previous one magnified by a power of ten. Galaxies, planets, lakes, DNA, atoms can obviously only be detected at the appropriate space scales. Similarly, in the experiments described in the previous sections 
different type of regularities (e.g. corners and long corridors) extend over different time scales (ranging from a few hundreds of milliseconds to several seconds) and can only be detected by neural processes integrating information at different time scales.

The results described in this paper demonstrate that robots provided with control systems that are suited to deal with amount of changes in amount of time can solve hard problems that require to integrate sensory-motor information over time at different time scales. Indeed, the self-localization problem described in the previous sections could only be solved by using artificial neurons in which: (1) the output changes at different rate according to genetically encoded parameters, and (2) the output is reset when the net input goes below a genetically encoded threshold (corresponding to the bias of the neuron). The former aspect seems to be crucial for detecting regularities occurring at different time scales. The analysis of the evolved individuals indeed showed that regularities occurring at short time scales (such us sensory-motor states experienced while the robot was negotiating a corner) were detected by neurons with fast changing rates while regularities occurring at longer time scales (such as sensory-motor states experienced while the robot was traveling along a long corridor) were detected by neurons with slow changing rates. The latter aspect seems to be crucial to detect events that last a given amount of time. In agents that are situated in a realistic environment, sensory and internal neurons provide information that extends over time and that does not have any meaning when isolated from time duration. From this point of view one might observe that reactive systems are a limit case in which regularities necessary to achieve the goal of the agent are visible at a single time scale which, at least roughly, corresponds to the time scale used to update the neurons of the agent. Finally, it should be noted that the same arguments that apply to the detection of regularities also apply to the effects that a neuron should have on other neurons or on actuators, i.e. the two characteristics described above might be crucial to produce effects occurring at different time scales and/or effects lasting a given amount of time. Overall, the obtained results show the importance of viewing cognition as the results of processes that unfolds in time at different time scales (van Gelder and Port, 1995).

Finally, the experiments presented in this paper demonstrate that the embodied cognitive science approach can tackle complex problems that necessarily require internal mechanisms. In his seminal work, Randall Beer showed how certain problems such us walking require agents provided with an internal dynamics (Beer, 1995). Moreover, it showed that evolved legged animats, controlled by continuous time recurrent neural networks, not only display an ability to produce an effective walking behavior on the basis of their internal dynamics but also display an ability to modulate their walking behavior on the basis of the sensory-motor flow, i.e. evolved networks displayed an ability to speed up or slow down the walking behavior when the rate of change of the sensors was artificially increased or decreased. This walking problem is a case in which changes in sensory states do not provide enough information to produce the appropriate motor actions and also an example of behavior that requires changes in motor actions that occur at a different (slower) time scale than changes occurring in sensory inputs (for other examples, see Ziemke 2000; Tuci, Harvey, and Quinn, 2002). In this paper we showed how a similar approach can be used to solve problems, such us self-localization, which do not only require to produce changes in motor states occurring at a different time scale from changes occurring in sensory states but that also require to deal with different time scales. Indeed, self-localizing robots are able to work at a rather fast time scale (in the order of $100 \mathrm{~ms}$ ) to detect obstacles and avoid them, at a slightly lower time scale (in the order or few hundreds of milliseconds) to detect regularities such us corners or narrow corridors, and at a even slower time scales (in the order of seconds) to detect high order regularities such as rooms and produce self-localization outputs. As in the case of the walking legged robots described above, the wheeled robots presented in this paper do not 
only have an internal dynamic but also have an ability to integrate their internal states with the sensory-motor flow in order to, for example, self-localize after being placed in a random selected position of the environment.

\section{Acknowledgements}

The author thanks Davide Marocco, Stefano Ghirlanda, Dario Floreano and Gianluca Baldassarre for the helpful discussions and insightful suggestions.

\section{References}

Beer R.D. (1995). A dynamical systems perspective on agent-environment interaction. Artificial Intelligence, 72:173-215.

Beer R.D. (1996). Toward the evolution of dynamical neural networks for minimally cognitive behavior. In P. Maes, M. Mataric, J. Meyer, J. Pollack and S. Wilson (Eds.), From animals to animats 4: Proceedings of the Fourth International Conference on Simulation of Adaptive Behavior. Cambridge, MA: MIT Press.

Beer R.D. and Gallagher J. C. (1992) Evolving dynamical neural networks for adaptive behavior. Adaptive Behavior, 1:91-122

Brooks R.A. (1991) Intelligence without reason. In J. Mylopoulos \& R. Reiter (Eds.), Proceedings of 12th International Joint Conference on Artificial Intelligence. San Mateo, CA: Morgan Kaufmann.

Clark A. (1997) Being There: Putting Brain, Body and World Together Again. Cambridge, MA: MIT Press.

Floreano, D. and Mattiussi, C. (2001) Evolution of Spiking Neural Controllers for Autonomous Vision-based Robots. In T. Gomi (ed.), Evolutionary Robotics IV, Berlin: Springer-Verlag.

Hopfield J.J. (1984). Neurons with graded response properties have collective computational properties like those of two-state neurons. Proceedings of the National Academy of Sciences of the United States of America, 81: 3088-3092.

Keijzer F. (2001). Representation and behavior. Cambridge, MA: MIT Press.

Maturana H.R. \& Varela F.J. (1980). Autopoiesis and cognition: the realization of the living. Dordrecht: reidel.

Maturana H.R. \& Varela F.J. (1987). The three of knowledge: the biological roots of human understanding. Boston: New Science Library.

Mondada R., Franzi E. \& Ienne P. (1993). Mobile robot miniaturization: A tool for investigation in control algorithms. In T.Y. Yoshikawa \& F. Miyazaki (Eds.), Proceedings of the Third International Symposium on Experimental Robots. Berlin, Springer-Verlag.

Nolfi S. (2000). Evorobot 1.1 User Manual. Technical Report. Rome: Institute of Psychology (available at http://gral.ip.rm.cnr.it/evorobot/simulator.html).

Nolfi S. (2002). Power and Limits of Reactive Agents. Neurocomputing, 42:119-145.

Nolfi S., Floreano D. (2000). Evolutionary Robotics. Cambridge, MA: MIT Press.

Nolfi S. \& Marocco D. (2001). Evolving robots able to integrate sensory-motor information over time. Theory in Biosciences, 120:287-310

Pfeifer R. and Scheier C. (1999) Understanding Intelligence. Cambridge, MA: MIT Press

Rieke F., Warland D., van Steveninck R. and Bialek W. (1997). Spikes. Exploring the Neural Code. Cambridge, MA: MIT Press. 
Shultz W., Apicella P., Romo R. \& Scarnati E. (1995). Context-dependent activity in primate striatum reflecting past and future behavioral events. In Houk J.C., Davis J.L \& Beiser D. (Eds.), Models of Information Processing in the Basal Ganglia. Cambridge, MA: MIT Press/Bradford Book.

Tuci E., Harvey I., and Quinn M. (2002). Evolving integrated controllers for autonomous learning robots using dynamic neural networks. In B. Hallam, D. Floreano, J. Hallam, G. Hayes, J-A. Meyer (Eds.) Proceedings of the VII International Conference on Simulation of Adaptive Behavior. Cambridge, MA: MIT Press.

Van Gelder T. and Port R.F. (1995). It's about time: an overview of the dynamical approach to cognition. In R.F. Port and T van Gelder (eds.) Minds as motion: exploration in the dynamics of cognition. Cambridge, MA: MIT Press.

Varela F.J., Rosch E., and Thompson E. (1991). The Embodied Mind: Cognitive Science and Human Experience. Cambridge, MA: MIT Press/Bradford Books.

Villa A. (2000). Empirical evidences about temporal structure in multi-agent recordings. In R. Miller (ed.), Time and the Brain. Reading, UK: Harwood Academic Publishers.

Ziemke (2000). On 'parts' and 'wholes' of adaptive behavior: Functional modularity and diachronic structure in recurrent neural robot controllers. In: J-A Meyer, A. Berthoz, D. Floreano, H.L. Roitblat, and S.W. Wilson (Dds.) From Animals to Animats 6. Proceedings of the VI International Conference on Simulation of Adaptive Behavior. Cambridge, MA: MIT Press. 